American Journal of Applied Mathematics
2020; 8(2): $51-63$
http://www.sciencepublishinggroup.com/j/ajam
doi: 10.11648 /j.ajam.20200802.12
ISSN: $2330-0043$ (Print); ISSN: $2330-006 \mathrm{X}$ (Online)

\title{
Medical Image Intelligent Recognition Predicts the Recessivity Variation of Human Tissue
}

\author{
Zhu Rongrong \\ Differential Incremental Equilibrium Geometry, Mathematics Research of HR, Fudan University, Shanghai, China
}

\author{
Email address: \\ rongrongzhu1969@163.com
}

\section{To cite this article:}

Zhu Rongrong. Medical Image Intelligent Recognition Predicts the Recessivity Variation of Human Tissue. American Journal of Applied Mathematics. Special Issue: Molecular Cellular Information Mathematics-Differential Incremental Equilibrium Geometry.

Vol. 8, No. 2, 2020, pp. 51-63. doi: 10.11648/j.ajam.20200802.12

Received: January 7, 2020; Accepted: February 10, 2020; Published: February 24, 2020

\begin{abstract}
By analyzing and predicting the latent variation of human tissues, the concept of iterative programming of heavy kernel clustering is introduced to solve the problem of intelligent recognition of medical images of inflammation and cancer. Inflammatory cells modify the accumulation of cancer cells and leap to the early stage of cancer, which is called image entropy. The hypercomplex symmetric structure of the edge sliding kernel of the entropy kernel of high-dimensional $s \geq 6$ image. As well as the fusion of image entropy nucleus dumbbell double sphere complex sphere, the exchangeability of the central source extreme compression line sink; the central source superstring sink compresses to the critical point, and the unconstrained $2 N+1$ laminated incision will cause the high-dimensional superstring sink to break up and release the exfoliated cells. Non analytic exploitation is the inverse kernel factor of aidicom that can judge the entropy of latent tissue variation image from inflammation to early cancer. It is a foundation of revealing (predicting) system recognition data array, and can carry the first-order and second-order partial differential carriers of kernel core area. In the medical image, the identification of inflammation and cancer often troubles doctors. Based on the inherent logic between cell modification fluctuation and image, aidicom system gives the concept of image entropy, and uses the dieg algorithm to complete the classification of focus detection and recognition, as well as the prediction of future development.
\end{abstract}

Keywords: Heavy Nuclear Clustering, Image Entropy, Cancer Cell Accumulation, Cell Modification,

Tissue Recessive Variation, Intelligent Recognition

\section{Introduction}

\subsection{DIEG Algorithm}

"Differential Incremental Equilibrium Geometry" is abbreviated as DIEG [1]. In this system, the first algorithm of DIEG is used, and the concept of iterative programming of heavy kernel clustering is introduced. The solution of intelligent recognition of medical image of inflammation and cancer, which is used to analyze and predict the latent variation of human tissue, is given.

\subsection{Image Recognition and Classification}

The traditional medical image recognition App is based on pixels or vector lines. Because the algorithm is old, it can only recognize visible tumors, so far it is difficult to reach the recognition level of image medical human experts. In view of the damage to human body caused by CT enhancement, CT Radiography and other inspection methods, AIDicom only detects lesions, outlines target areas, biometrics and grading on plain CT scan images, so as to minimize the impact on human body, and finally accurately predict the future development of lesions.

\section{Medical Image Entropy}

\subsection{The Concept of Medical Image Entropy}

Differential incremental equilibrium geometry predicts the growth trend of bud nucleus vibration kernel shadow factor [2], has the entropy (s) of kernel evolution data array to predict the second-order tumor risk of inflammatory cells modified to the medical image entropy (s) of cancer cell accumulation [3]. 


\subsection{Similar to the Expansion of the Multi Variable Taylor Expansion Formula, the Corner Points of the Abnormal Points of Medical Images Are Obtained}

It has the robust phenomenon of corner edge, realizes the integrity of the core matrix transformation aidicom system, multifunctional, multimodal, robust and other intelligent digital analog AI system [4].

$$
\begin{aligned}
\Sigma \Delta S & =S=\Sigma\left[\left(\sum_{i=2}^{m} \frac{i A_{i}}{2}\right)^{\Delta t-1}+\left(\sum_{i=2}^{m} \frac{i B_{i}}{2}\right)^{\Delta t-1}\right]^{2} \\
& =\Sigma\left[\left(w_{(u, v)}^{\Delta t-1}\right)^{2}+2 w_{(u, v)}^{\Delta t-1} w_{\left(u^{\prime}, v^{\prime}\right)}^{\Delta t-1}+\left(w_{\left(u^{\prime}, v^{\prime}\right)}^{\Delta t-1}\right)^{2}\right] \\
& =\left[u^{\prime}, v^{\prime}\right]\left[\begin{array}{ll}
\lambda_{1} & \\
& \lambda_{2}
\end{array}\right]\left[\begin{array}{l}
u_{v^{\prime}}^{\prime} \\
v^{\prime}
\end{array}\right.
\end{aligned}
$$

Three dimensional reconstruction of non-linear edge focus, detection corner function of abnormal corner of medical image, and geometric morphology of super three-dimensional convex structure. The high-dimensional space of three-dimensional focus with nonlinear edge of super three-dimensional convex shape, $N^{\prime}$ is the changed matrix.

Super three-dimensional convex shape of nonlinear edge of three-dimensional lesions in high-dimensional space. $\mathrm{N}^{\prime}$ is the changed matrix, Patient 3[N_13799].

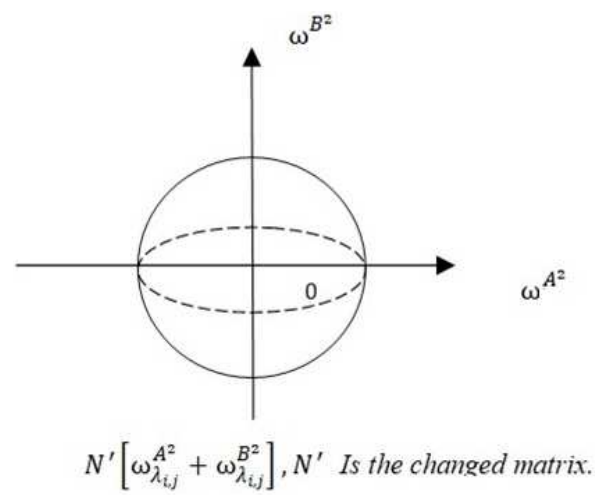

Figure 1. Matrix of standard three-dimensional space.

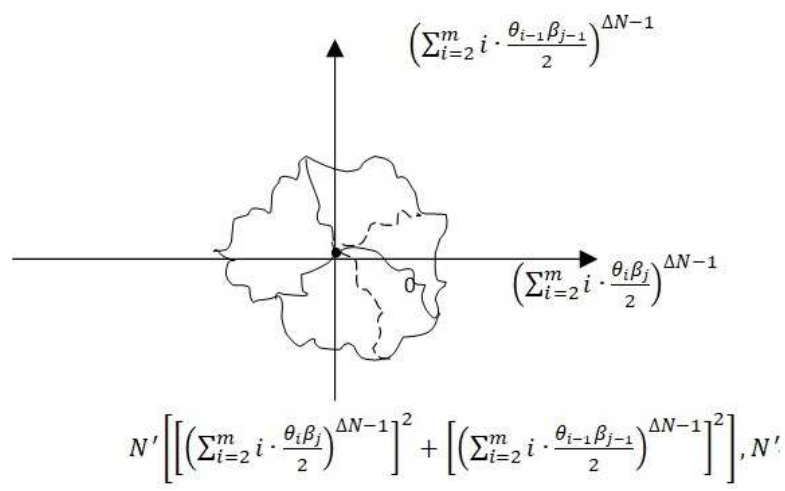

Figure 2. The super $3 d$ convex shape of the marginal lesions.

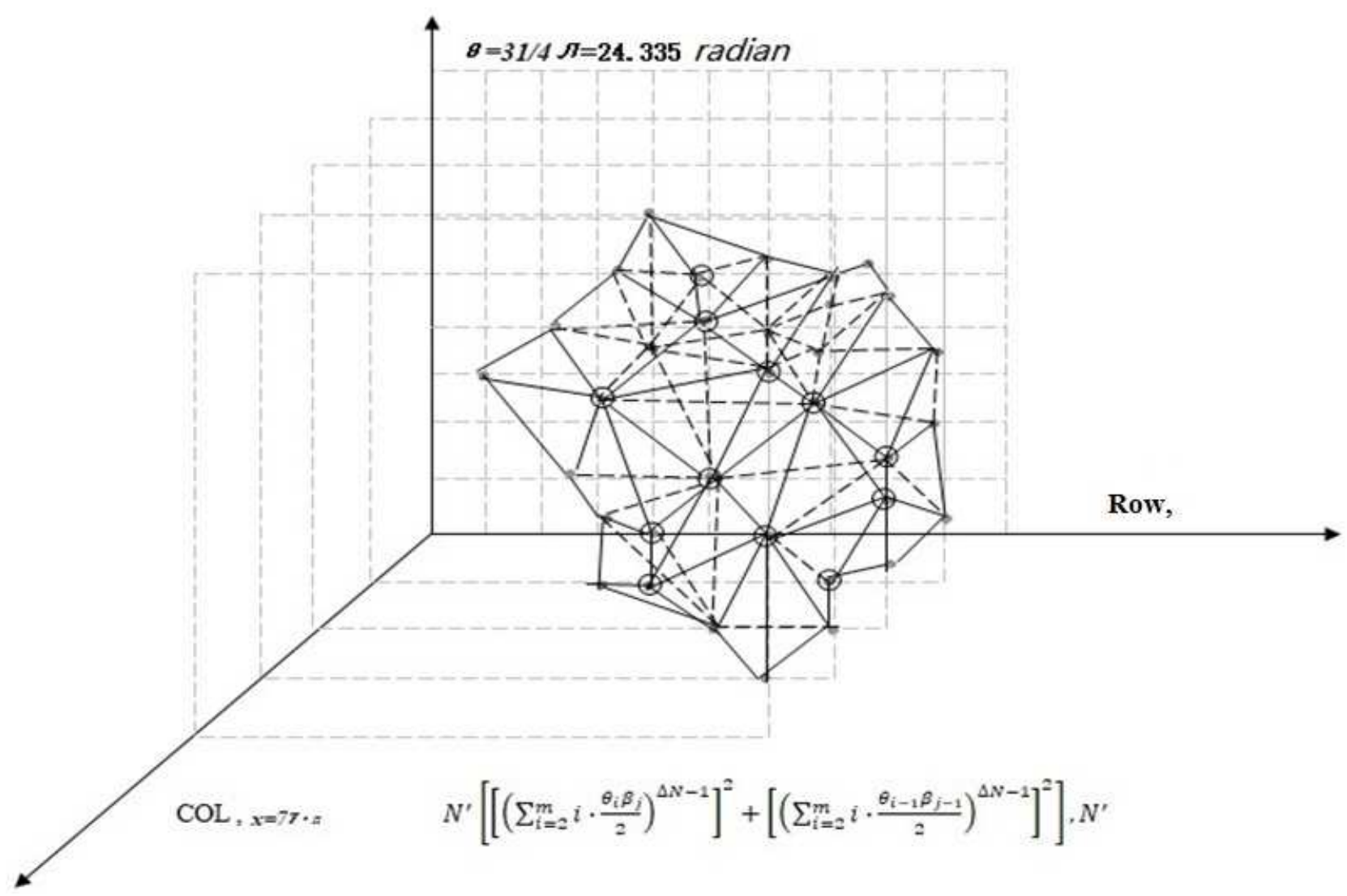

Figure 3. Nonlinear edge three-dimensional focus high-dimensional real space. 


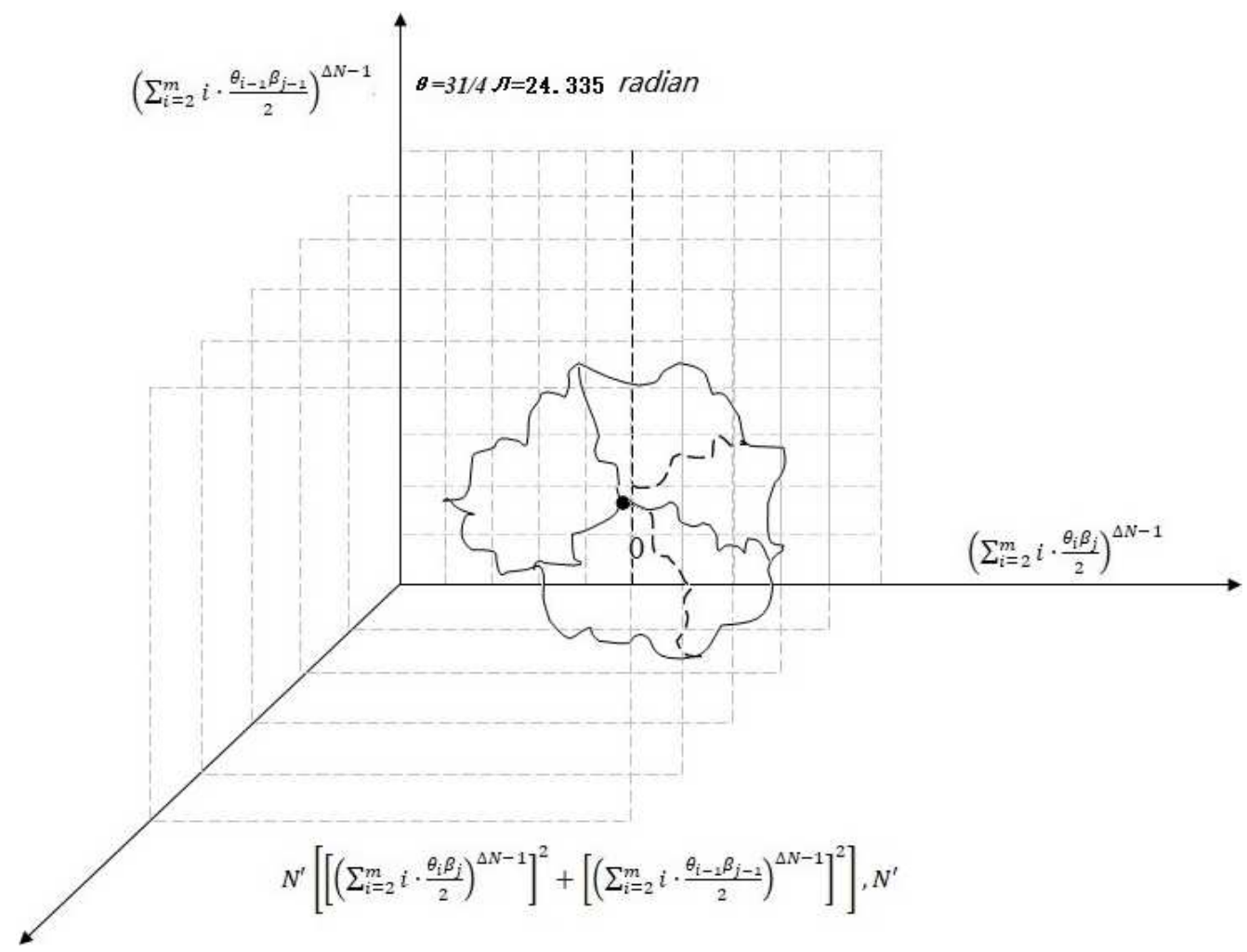

Figure 4. Nonlinear edge three dimensional focus high dimensional abstract space.

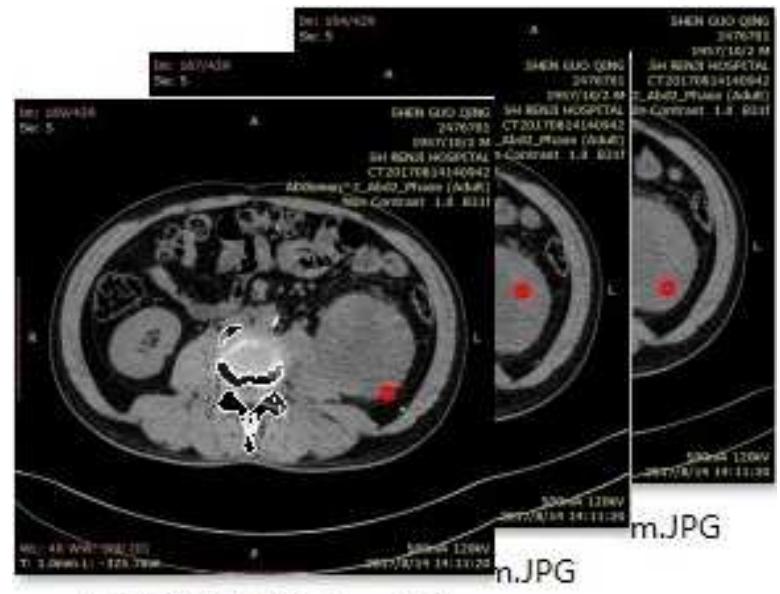

IMG-0089 169.dcm.JPG

Figure 5. Images of non-linear edge focus and 4 images of rotation (31/4) $\pi$.

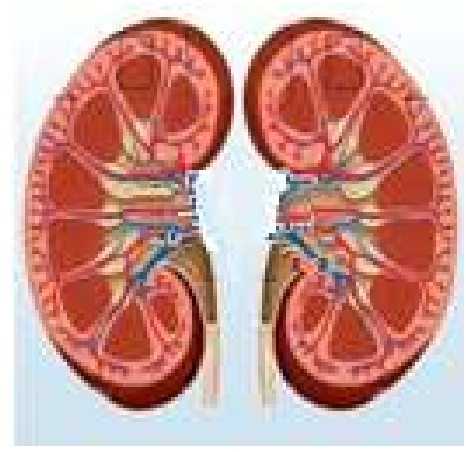

Figure 6. The real space of three-dimensional lesions of renal carcinoma.

\subsection{Weak Nonlinear Finite Isopotential Hypercircle- Hypercomplex Hypercircle Image Abnormal Target Region}

The smooth edge of the medical image area is fine sketched.

$$
\sum \Delta \mathrm{S}=S=\sum\left[\left(\sum_{i=2}^{m} \frac{i A_{i}}{2}\right)^{\Delta t-1}+\left(\sum_{i=2}^{m} \frac{i B_{i}}{2}\right)^{\Delta t-1}\right]^{2}
$$

The area edge sketch of medical image tissue with finite weak nonlinear equal potential difference is in essence the abnormal target area of hypercomplex and hypercircle images [5]. The complex structure from normal cell modification to cancer cell modification, no matter from the geometric topology construction of cell modified microstructure or from the three-dimensional construction of image tissue target area, has the conceptual equivalence and the similarity in mathematical deduction.

$$
\begin{array}{r}
\int \sum\left({ }_{\mathrm{m}} \mathrm{S}_{\text {First }}^{\text {Sum }}\right)^{\nabla}\left(A_{i}(\lambda), B_{i}\left(\lambda^{\prime}\right)\right) \\
\cong \sum\left({ }_{\mathrm{m}} \mathrm{S}_{\text {First }}^{\text {Sum }}\right)_{\left(A_{i}(\lambda), B_{i}\left(\lambda^{\prime}\right)\right)} \cong \\
{\left[\left(\sum_{i, j=2}^{m} i \cdot \frac{\Delta A_{i j}^{2}}{2}\right)_{M I N}^{\Delta t-1}-\left(\sum_{i, j=2}^{m} i \cdot \frac{\Delta B_{i j}^{2}}{2}\right)_{M A X}^{\Delta t-1}\right]}
\end{array}
$$

The edge of the area of the tissue abnormal target in medical image is delineated by finite weak nonlinear equipotential difference. Because of the use of integration with gradient edge, the area edge of tissue abnormal target area is more smooth, in fact, it has super complex and super circular image abnormal target area. The corner of abnormal point in medical image has the robust phenomenon of corner edge. 


$$
N\left[\begin{array}{cc}
\omega_{\lambda_{i}}^{A} & \\
& \omega_{\lambda_{j}}^{A}
\end{array}\right] \circ N\left[\begin{array}{ll}
\omega_{\lambda_{i}}^{B} & \\
& \omega_{\lambda_{j}}^{B}
\end{array}\right]^{\mathrm{T}}=N^{\prime}\left[\left[\left(\sum_{i, j=2}^{m} i \cdot \frac{\theta_{i} \beta_{j}}{2}\right)^{\Delta N-1}\right]^{2}+\left[\left(\sum_{i, j=2}^{m} i \cdot \frac{\theta_{i-1} \beta_{j-1}}{2}\right)^{\Delta N-1}\right]^{2}\right]
$$

The abnormal target areas of three-dimensional or high-dimensional images of hypercomplex and hypercircle [6].

$$
\sum\left({ }_{\mathrm{m}} \mathrm{S}_{\text {First }}^{\text {Sum }}\right)_{\left(A_{i}(\lambda), B_{i}\left(\lambda^{\prime}\right)\right)} \cong\left[\left(\sum_{i, j=2}^{m} \frac{\Delta A_{i j}^{2}}{2}\right)_{\lambda}^{\Delta t-1}-\left(\sum_{i, j=2}^{m} i \cdot \frac{\Delta B_{i j}^{2}}{2}\right)_{\lambda^{\prime}}^{\Delta t-1}\right]
$$

The matrix transformation of $N \rightarrow N^{\prime}$ and the three-dimensional or higher dimensions of the super circle can solve the variation target area of the three-dimensional spatial rotation of the focus in the medical image recessive tissue.

$$
N\left[\begin{array}{cc}
\theta_{i j}(\lambda) & \\
& \theta_{(i-1)(j)}(\lambda)
\end{array}\right] \circ N\left[\begin{array}{ll}
\beta_{i j}\left(\lambda^{\prime}\right) & \\
& \beta_{(i-1)(j)}\left(\lambda^{\prime}\right)
\end{array}\right]^{T}=N^{\prime}\left[\left(\sum_{i, j=2}^{m} \frac{\Delta \theta_{i}^{\lambda} \beta_{j}^{\lambda}}{2}\right)^{\Delta N-1}+\left(\sum_{i=2}^{m} i \cdot \frac{\Delta \theta_{i}^{\lambda^{\prime}} \beta_{j}^{\lambda^{\prime}}}{2}\right)^{\Delta N-1}\right]
$$

\subsection{Hyperspace Transformation of Image Entropy of Sparse Matrix}

The sparse matrix $M \rightarrow M^{\prime}$ super complex transformation, and the image entropy Super Space Transformation of the recessive mutation target domain of high-dimensional medical image organization [7]. The depth statistical joint clustering kernel of sparse matrix transformation has the characteristics of hypercomplex and high-dimensional image abnormal target domain.

$$
\begin{aligned}
& M\left[\begin{array}{ccc}
\theta_{i j} & \Delta_{\theta_{x}} & \\
\theta_{i(j+1)} & & \theta_{n m} \\
& \Delta_{\theta_{y}} & \theta_{n(j+m)}
\end{array}\right]{ }^{\circ} M^{\prime}\left[\begin{array}{ccc} 
& \Delta_{\beta_{x}} & \beta_{n m} \\
\beta_{i j} & & \beta_{n(j+m)} \\
\beta_{i(j+1)} & \Delta_{\beta_{y}}^{T} &
\end{array}\right] \\
& =N^{\prime}\left[\left[\begin{array}{ccc}
A_{i j} & \cdots & \\
\vdots & \ddots & \vdots \\
& \cdots & A_{(i+n)(j+m)}
\end{array}\right]\left[\begin{array}{l}
\lambda_{1} \\
I
\end{array}\right]^{\Delta_{x,(x+1)}^{2}}+\left[\begin{array}{ll}
\lambda_{2} & \\
& I
\end{array}\right]^{\Delta_{y,(y+1)}^{2}}\left[\begin{array}{ccc}
B_{i j} & \cdots & \\
\vdots & \ddots & \vdots \\
& \cdots & B_{(i+n)(j+m)}
\end{array}\right]\right]
\end{aligned}
$$

Sparse matrix joint clustering the abnormal target domain of hyper complex kernel $\left(\Delta_{\theta_{x}}, \Delta_{\theta x+1}\right)$ and $\left(\Delta_{\beta}{ }^{T}, \Delta_{\beta}{ }^{T}{ }_{y+1}\right)$ under angular. To high-dimensional image. The disturbance of medical image data matrix is checked by the reduced dimension edge sliding target domain of the hypercomplex high-dimensional image. The data atlas of sparse matrix and joint clustering kernel is of sparse matrix combined with clustering kernel, which is called complex dynamic system [8].

$$
\begin{aligned}
& M\left[\begin{array}{ccc}
\theta_{i j} & \Delta_{\theta_{x}} & \\
\theta_{i(j+1)} & & \theta_{n m} \\
& \Delta_{\theta_{y}} & \theta_{n(j+m)}
\end{array}\right] M^{\prime}\left[\begin{array}{ccc} 
& \Delta_{\beta_{x}}^{T} & \beta_{n m} \\
\beta_{i j} & & \beta_{n(j+m)} \\
\beta_{i(j+1)} & \Delta_{\beta_{y}}^{T} &
\end{array}\right]\left[\theta^{\nabla}(x, y), i \cdot \beta^{\nabla}(x, y)\right] \cdot \vec{n} \\
& =\vec{n} \cdot\left[\begin{array}{cc}
O_{(x, y)}^{\nabla} M^{\prime} & -\frac{1}{2} \cdot d_{(x, y)}^{\beta} d^{2} \theta_{(x, y)} \beta_{n(j+m)} \theta\left(x^{2}, y\right) \\
\frac{1}{2} i \cdot d_{(x, y)}^{\theta} d^{2} \beta_{(x, y)} \theta_{i(j+1)} \beta\left(x, y^{2}\right) & O_{(x, y)}^{\nabla} M
\end{array}\right]
\end{aligned}
$$

\subsection{Covariance Kernel Complex Ellipse}

The covariance kernel complex ellipse is the inverse kernel perturbation kernel of the covariance matrix variable of the image entropy kernel which constrains the morphological distribution.

$$
\Omega^{-1}\left(C_{(x, y)}^{2}\right)=s \cdot\left[\frac{\lambda_{11} \times \ln \left(\theta_{(x, y)}^{2}\right) \cdot \cos \theta_{x}}{\ln \left(\vartheta_{11} \cdot \operatorname{Cos} \theta_{(\alpha, x)}^{2}, \vartheta_{12} \cdot \sin \beta_{(\alpha, y)}^{2}\right)_{C^{2}}}-\frac{\lambda_{12} \times \ln \left(\beta_{(x, y)}^{2}\right) \cdot \sin \beta_{y}}{\ln \left(\vartheta_{11} \cdot \operatorname{Cos} \theta_{(\alpha, x)}^{2}, i \cdot \vartheta_{12} \cdot \sin \beta_{(\alpha, y)}^{2}\right)_{C^{2}}}\right]
$$

and $\left(\vartheta_{11} \cdot \operatorname{Cos} \theta_{(\alpha, x)}^{2}, i \cdot \vartheta_{12} \cdot \operatorname{Sin} \beta_{(\alpha, y)}^{2}\right)_{C^{2}}$ complex ellipse $\lambda_{11}, \lambda_{12}$.

representing covariance kernel, $\mathrm{s}$ represented dimension,

is the real parameter of matrix, $\vartheta_{11}, \vartheta_{12}$ is the real parameter 
of complex variable ellipse, $\theta_{(\alpha, x)} \rightarrow \theta_{\alpha}, \beta_{(\alpha, y)} \rightarrow \beta_{\alpha}$

is the covariance sparse matrix.

Compared with the cell modification from inflammation to cancer, "constrained hyperelliptical modification - image entropy reverse internal disturbing nucleus morphology distribution" and "non analytical exploration - recessive tissue variation image entropy reverse nuclear factor", the exfoliated cells expanded the constrained cell modification, which made the inflammation jump to cancer cell modification, and had the process of supersymmetric geometric morphology evolution.

The root of the inverse kernel solution of the covariance matrix variable of the image entropy core that constrains the shape distribution can correctly reveal the internal relationship of the covariance matrix variable, so as to correctly obtain the sliding kernel trend of the image entropy core that constrains the shape distribution, that is, the internal law of the development trend from inflammation to cancer, the hidden variation of human tissue, and the solution to block its development. According to the shape kernel of the hypercomplex symmetric structure of the edge sliding kernel of the entropy kernel of the high-dimensional $s \geq 6$ image.

Because $\theta_{\alpha}^{1}, \theta_{\alpha}^{2} \neq 0, \Omega_{\text {Entropykernel }}^{s \geq 6}\left(O_{i \cdot M \otimes M^{\prime}}^{\alpha \cdot \vec{n}}\right) \rightarrow 0$, i.e. image entropy or image entropy nucleus tends to zero, inflammation will jump to cancer, and inflammation of human tissue cannot be repaired, i.e. $\Omega^{-1}\left(C_{(x, y)}^{2}\right)$ divergence.

$$
\begin{aligned}
& \Omega_{\text {Entropy kernel }}^{s \geq 6}\left(O_{i \cdot M \otimes M^{\prime}}^{\alpha \cdot \vec{n}}\right)=i \cdot\left[\frac{1}{s} \cdot\left(\frac{\operatorname{Ln}\left(\vartheta_{11} \cdot \operatorname{Cos}\left(\theta_{1}^{x}+\theta_{2}^{x}\right)^{2}, i \cdot \vartheta_{12} \cdot \operatorname{Sin}\left(\beta_{1}^{y}+\beta_{2}^{y}\right)^{2}\right)}{\lambda_{11} \times \ln \left(\theta_{(x, y)}{ }^{2}\right) \cdot \operatorname{Cos} \theta_{x}-\lambda_{12} \times \ln \left(\beta_{(x, y)}{ }^{2}\right) \cdot \operatorname{Sin} \beta_{y}}\right)\right] \cdot d^{6}\left(\operatorname{Cos}\left(\theta_{x}^{2}\right) \otimes \operatorname{Sin}\left(\beta_{y}^{2}\right)\right), \\
& \left(\vartheta_{11} \cdot \operatorname{Cos}\left(\theta_{1}^{x}+\theta_{2}^{x}\right)^{2}, i \cdot \vartheta_{12} \cdot \operatorname{Sin}\left(\beta_{1}^{y}+\beta_{2}^{y}\right)^{2}\right)=1, \text { complex super circle, } \vartheta_{11}, \vartheta_{12} \equiv 1, \Omega_{\text {Entropy kernel }}^{s \geq 6}\left(O_{i \cdot M \otimes M^{\prime}}^{\alpha \cdot \vec{n}}\right) \rightarrow 0
\end{aligned}
$$

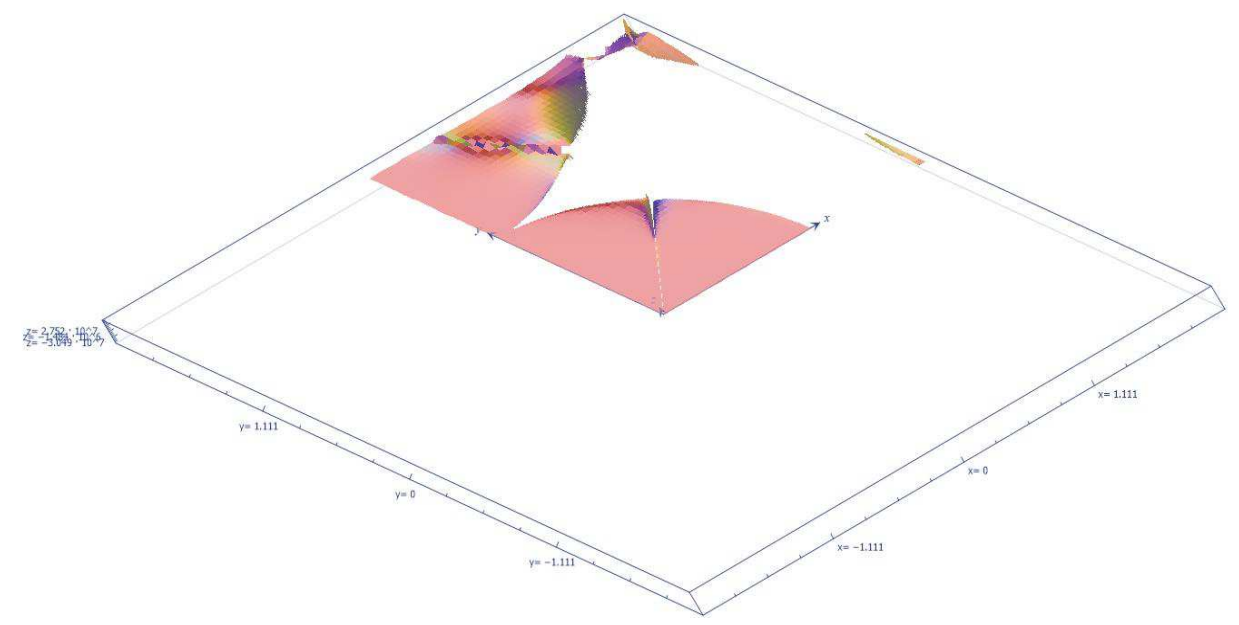

Figure 7. The morphological kernel of the hypercomplex symmetric structure of the edge sliding kernel of the entropy kernel of the high-dimensional $s \geq$ 6image.

On the constrained complex hypercircles.

$$
\begin{gathered}
\operatorname{Ln}\left(\vartheta_{11} \cdot \operatorname{Cos}\left(\theta_{1}^{x}+\theta_{2}^{x}\right)^{2}, i \cdot \vartheta_{12} \cdot \operatorname{Sin}\left(\beta_{1}^{y}+\beta_{2}^{y}\right)^{2}\right) \\
\text { if }\left(\vartheta_{11} \cdot \operatorname{Cos}\left(\theta_{1}^{x}+\theta_{2}^{x}\right)^{2}, i \cdot \vartheta_{12} \cdot \operatorname{Sin}\left(\beta_{1}^{y}+\beta_{2}^{y}\right)^{2}\right)=1, \text { complex super circle, } \vartheta_{11}, \vartheta_{12} \equiv 1
\end{gathered}
$$

\subsection{Construction of the Complex Dynamic Perturbation Kernel of the Hypercomplex and Circularity of the Constrained Laminated Notch of the Image Entropy Sliding Kernel}

We need to increase the dimension of its complex hypercircle.

$$
\begin{gathered}
\left(\vartheta_{11} \cdot \operatorname{Cos}\left(\theta_{1}^{x}+\theta_{2}^{x}\right)^{2 \cdot \frac{p}{q}}, i \cdot \frac{p}{q} \cdot \vartheta_{12} \cdot \operatorname{Sin}\left(\beta_{1}^{y}+\beta_{2}^{y}\right)^{2 \cdot \frac{p}{q}}\right) \\
\Omega_{\text {Entropy kernel }}^{S \geq 6}\left(O_{i p \cdot M \otimes M^{\prime}}^{\alpha \cdot \vec{n}}\right)=i \cdot p\left[\frac{1}{s} \cdot\left(\frac{\operatorname{Ln}\left(\vartheta_{11} \cdot \operatorname{Cos}\left(\theta_{1}^{x}+\theta_{2}^{x}\right)^{2} \cdot \frac{p}{q}, i \cdot \frac{p}{q} \cdot \vartheta_{12} \cdot \operatorname{Sin}\left(\beta_{1}^{y}+\beta_{2}^{y}\right)^{2} \cdot \frac{p}{q}\right)}{\left.\lambda_{11} \times \ln \left(\theta_{(x, y)}\right) \cdot \cos \theta_{x}-\lambda_{12} \times \ln \left(\beta_{(x, y)}\right)^{2}\right) \cdot \operatorname{Sin} \beta_{y}}\right)\right]^{*} d^{6}\left(\operatorname{Cos}\left(\theta_{x}^{2}\right) \otimes \operatorname{Sin}\left(\beta_{y}^{2}\right)\right)
\end{gathered}
$$

From the high-dimensional image entropy sliding core constraining $2 n$ lamination incision hyperconvexation to the image entropy sliding core non constraining $2 n$ lamination incision hyperconvexation [9]. $\left(D N A_{\text {Viruses }}, \mathrm{s}=10\right)$ 


$$
\begin{gathered}
\Omega_{\text {Entropykernel }}^{s=10}\left(O_{i p \cdot M \otimes M^{\prime}}^{\alpha \cdot \vec{n}}\right) \\
=i \cdot C_{s} \cdot \sum_{K=4}^{N}\left[\left(\frac{\left(\vartheta_{11} \cdot \operatorname{Cos}\left(\theta_{1}^{x}+\theta_{2}^{x}\right)^{2 \cdot \frac{p}{q}}, i \cdot \frac{p}{q} \cdot \vartheta_{12} \cdot \operatorname{Sin}\left(\beta_{1}^{y}+\beta_{2}^{y}\right)^{2 \cdot \frac{p}{q}}\right)}{\lambda_{11} \cdot C_{s 1} \cdot \theta_{(x, y)}{ }^{2} \cdot \operatorname{Cos} \theta_{x}-\lambda_{12} \cdot C_{s 2} \cdot \beta_{(x, y)}{ }^{2} \cdot \operatorname{Sin} \beta_{y}}\right)\right. \\
d^{2(K-1)}\left(\operatorname{Cos}\left(\theta_{x}^{K-2}\right) \otimes \operatorname{Sin}\left(\beta_{y}^{K-2}\right)\right) \\
\text { and } s=10 ; p, q=2,4,6, \ldots, 2 n
\end{gathered}
$$

When $D N A_{\text {virus }}$ embedded replication is complete, $s \geq 10$, if $s=11$ then

$$
\begin{gathered}
\Omega_{\text {Entropy kernel }}^{s=11}\left(O_{i p \cdot M \otimes M^{\prime}}^{\alpha \cdot \vec{n}}\right) \\
=i \cdot C_{s} \cdot \sum_{K=4}^{N}\left[\left(\frac{\left(\vartheta_{11} \cdot \operatorname{Cos}\left(\theta_{1}^{x}+\theta_{2}^{x}\right)^{2 \nabla \cdot \frac{p}{q}, i} \cdot \frac{p}{q} \cdot \vartheta_{12} \cdot \operatorname{Sin}\left(\beta_{1}^{y}+\beta_{2}^{y}\right)^{2 \nabla \cdot \frac{p}{q}}\right)}{\lambda_{11} \cdot C_{s 1} \cdot\left(\theta_{(x, y)}{ }^{2} \cdot \operatorname{Cos} \theta_{x}\right)^{\nabla}-\lambda_{12} \cdot C_{s 2} \cdot\left(\beta_{(x, y)}{ }^{2} \cdot \operatorname{Sin} \beta_{y}\right)^{\nabla}}\right)\right]^{*} . \\
d^{2 K-1}\left(\operatorname{Cos}\left(\theta_{x}^{K-2}\right) \otimes \operatorname{Sin}\left(\beta_{y}^{K-2}\right)\right), \\
\text { and } s=11 ; p, q=2,4,6, \ldots, 2 n
\end{gathered}
$$

When DNA damage is caused by even order transition of image entropy nucleus, it forms "the similar nuclear fission of complex dynamic perturbation nucleus with hyperconvexation and roundness of unrestricted $2 N$ lamination notch of image entropy sliding nucleus". The migration of $2 N+1$ lamination notch of image entropy gliding nucleus is $\pi / 4$ hyperconvexed and complex dynamic perturbation nucleus fission like.

$$
\begin{gathered}
\Omega_{\text {Entropy kernel }}^{s=11}\left(O_{i p \cdot M \otimes M^{\prime}}^{\alpha \cdot \vec{n}}\right) \\
=i \cdot 2 C_{s} \cdot \frac{p}{q} \cdot \sum_{K=4}^{N}\left[\left(\frac{\left(\vartheta_{11} \cdot \operatorname{Cos}\left(i^{j} \sum_{i=1}^{2 N+1} i \cdot \theta_{j}^{x}\right), i \cdot \vartheta_{12} \cdot \operatorname{Sin}\left(i^{j} \sum_{i=2}^{2 N} i \cdot \beta_{j}^{y}\right)\right)}{\lambda_{11} \cdot C_{s 1} \cdot\left(\theta_{(x, y)}^{2} \cdot \operatorname{Cos} \theta_{x}\right)^{\nabla}-\lambda_{12} \cdot C_{s 2} \cdot\left(\beta_{(x, y)}{ }^{2} \cdot \operatorname{Sin} \beta_{y}\right)^{\nabla}}\right)\right]^{*} \\
d^{2 K-1}\left(\operatorname{Cos}\left(\theta_{x}^{K-2}\right) \otimes \operatorname{Sin}\left(\beta_{y}^{K-2}\right)\right), \\
\text { and } s=11 ; p, q=2,4,6, \ldots, 2 n
\end{gathered}
$$

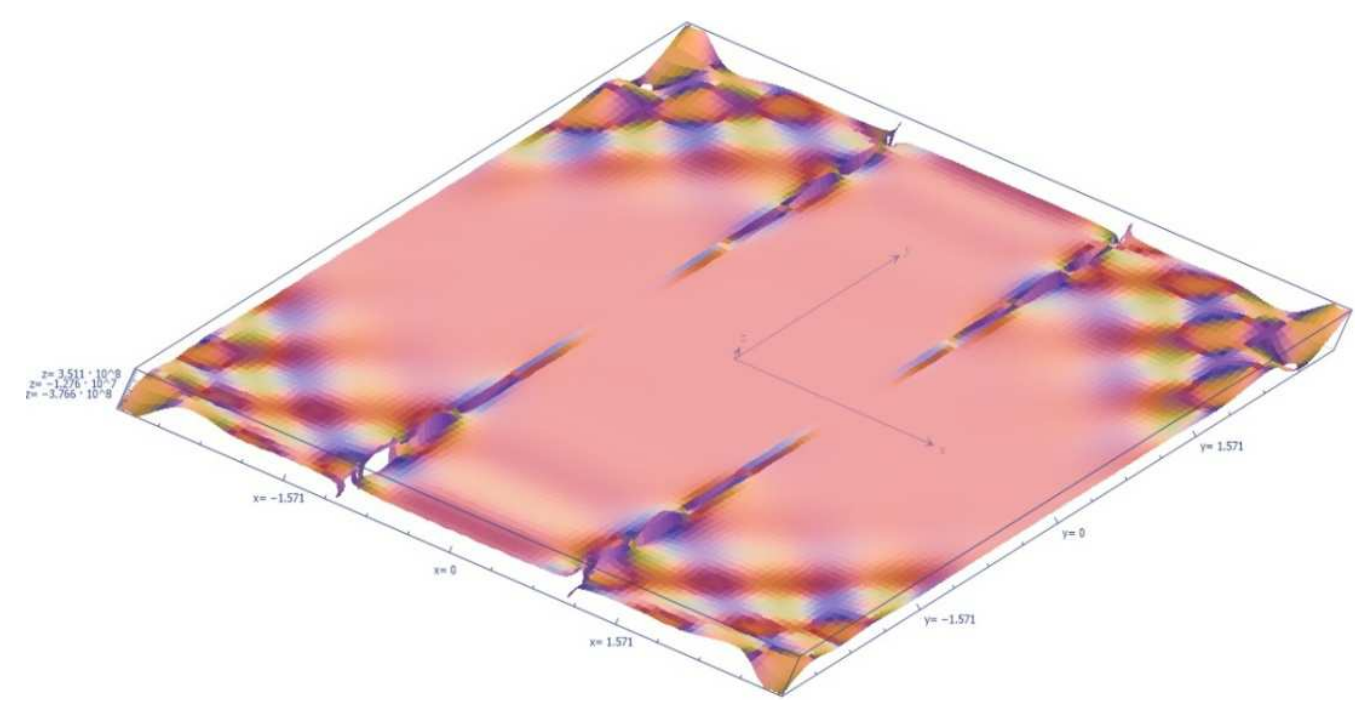

Figure 8. Complex dynamic perturbation nucleus with hyperconvexation and roundness of unrestricted $2 N$ lamination notch of image entropy sliding nucleus. 
It shows the importance of the quasi thinking iterative programming of the heavy kernel clustering (single clustering and joint clustering) in aidicom image recognition and the nuclear fission like depth fusion of the migration $\pi / 4$ of $2 N+1$ stack notch of the first order partial differential of the unconstrained sliding kernel of image entropy. Heavy kernel clustering (single clustering, joint clustering) quasi thinking iterative programming-homomorphic kernel exchangeability of hyperconvexation-image entropy sliding kernel unconstrained first-order partial differential $2 N+1$ stacking notch migration $\pi / 4$ hyperconvexation complex dynamic perturbation kernel fission [10].

$$
\begin{aligned}
& \Omega_{\begin{array}{c}
\text { Entropy kernel } \\
\text { embedded clustering }
\end{array}}^{S=11}\left(O_{i p \cdot M \otimes M^{\prime}}^{\alpha \cdot \vec{n}}\right) \\
& =i \cdot 2 C_{s} \cdot \frac{p}{q} \cdot \sum_{K=4}^{N}\left[\left(\frac{\left(\vartheta_{11} \cdot \operatorname{Cos}\left(i^{j} \sum_{i=2}^{2 N} i \cdot \theta_{j}^{x}\right), i \cdot \vartheta_{12} \cdot \operatorname{Sin}\left(i^{j} \sum_{i=1}^{2 N+1} i \cdot \beta_{j}^{y}\right)\right.}{\lambda_{11} \cdot C_{s 1} \cdot\left(\theta_{(x, y)}{ }^{2} \cdot \operatorname{Cos} \theta_{x}\right)^{\nabla}-\lambda_{12} \cdot C_{s 2} \cdot\left(\beta_{(x, y)}{ }^{2} \cdot \operatorname{Sin} \beta_{y}\right)^{\nabla}}\right)\right]^{*} \cdot \cdot d^{2 K-1}\left(\operatorname{Cos}\left(\theta_{x}^{K-2}\right) \otimes \operatorname{Sin}\left(\beta_{y}^{K-2}\right)\right), \\
& \text { and } s=11 ; p, q=2,4,6, \ldots, 2 n \\
& \text { (E) }
\end{aligned}
$$

Figure 9. Image entropy sliding kernel is unconstrained. The first order partial differential $2(2 N+1)$ laminated notch complex dynamic perturbation kernel.

"The homomorphic kernel of the logical split structure of double hypercomplex and circular transformation is exchangeable. The image entropy sliding kernel is unconstrained. The first order partial differential $2(2 N+1)$ laminated notch complex dynamic perturbation kernel" is a set of core equations [11].

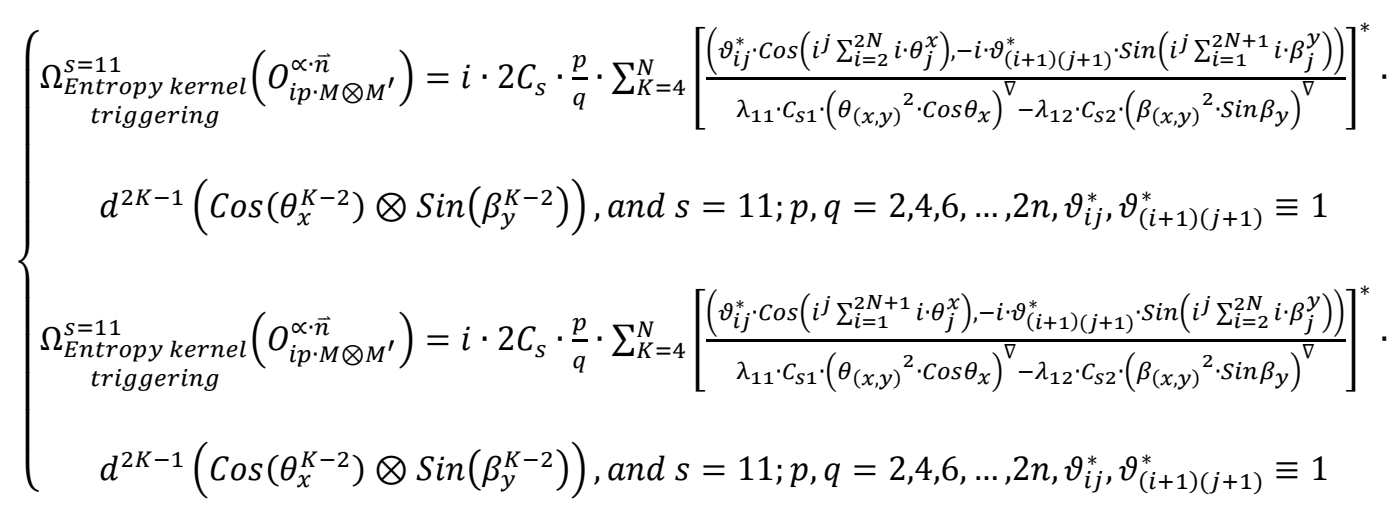

The deep feature information and the commutativity of homomorphic kernel of complex variable circle w logic split structure form complex variable dynamics similar to two camera sources [12].

$$
\begin{gathered}
{\left[w(A), w\left(A^{*}\right)\right] \cong\left(\frac{1}{4}\right)^{n}\left[\operatorname{Sin}\left(A_{1}+\sum_{i=2}^{m} A_{i}+n \cdot \frac{\pi}{4}\right)+\operatorname{Sin}\left(A_{1}^{*}-\sum_{i=2}^{m} A_{i}^{*}+n \cdot \frac{\pi}{4}\right)\right]^{n-1} \otimes\left(\Delta_{A}^{n}, \Delta_{A^{*}}^{n}\right), \text { and }} \\
\text { if }\left(\Delta_{A}^{n}, \Delta_{A^{*}}^{n}\right) \equiv 1, w(A) \sim w\left(A^{*}\right)
\end{gathered}
$$


$\left(\sqrt{\operatorname{Cos}^{n}(A)}, i \cdot \sqrt{\operatorname{Sin}^{n}\left(A^{*}\right)}\right) \equiv 1, n=4 \quad$ is $\quad$ a high dimensional complex circle with non robustness removed. So $\left[w(A), w\left(A^{*}\right)\right]$ is the dimension reduction (planarization) processing of robust sliding angle depth feature information with corner points. Depth feature information $2 D$ dynamic corner complex circle logic split homomorphic kernel. $\left[w(A), w\left(A^{*}\right)\right]$ is the dimension reduction (planarization) processing of depth feature information of robust sliding angle with corner.

\section{The Nonlinear Estimation Interval of the Central Limit Theorem of the Normal Distribution of Joint Clustering}

Clustering (joint clustering) and weak robustness of discrete gradient data clustering the nonlinear estimation interval formula of the central limit theorem, a normal distribution for extracting the feature information of sliding angle depth [13].

$$
\begin{aligned}
& \qquad\left[w(A), w\left(A^{*}\right)\right] \cong\left(\frac{1}{4}\right)^{n}\left[\operatorname{Sin}\left(A_{1}+\sum_{i=2}^{m} A_{i}+n \cdot \frac{\pi}{4}\right)+\operatorname{Sin}\left(A_{1}^{*}-\sum_{i=2}^{m} A_{i}^{*}+n \cdot \frac{\pi}{4}\right)\right]^{n-1} \otimes\left(\sqrt{\operatorname{Cos}^{n}(A)}, i \cdot \sqrt{\operatorname{Sin}^{n}\left(A^{*}\right)}\right), \\
& \text { and Central limit estimation interval }\left[\frac{1}{4} \operatorname{Sin}\left(\left(P\left(\bar{X}-\frac{\sigma_{\circ}}{\sqrt{n}} \mu_{1-\frac{\alpha}{2}}\right)+\frac{\pi}{4}\right)+2 k \pi\right), \frac{1}{4} \operatorname{Sin}\left(\left(P\left(\bar{X}+\frac{\sigma_{\circ}}{\sqrt{n}} \mu_{1-\frac{\alpha}{2}}\right)+\frac{\pi}{4}\right)+2 k \pi\right)\right], n=4 p
\end{aligned}
$$

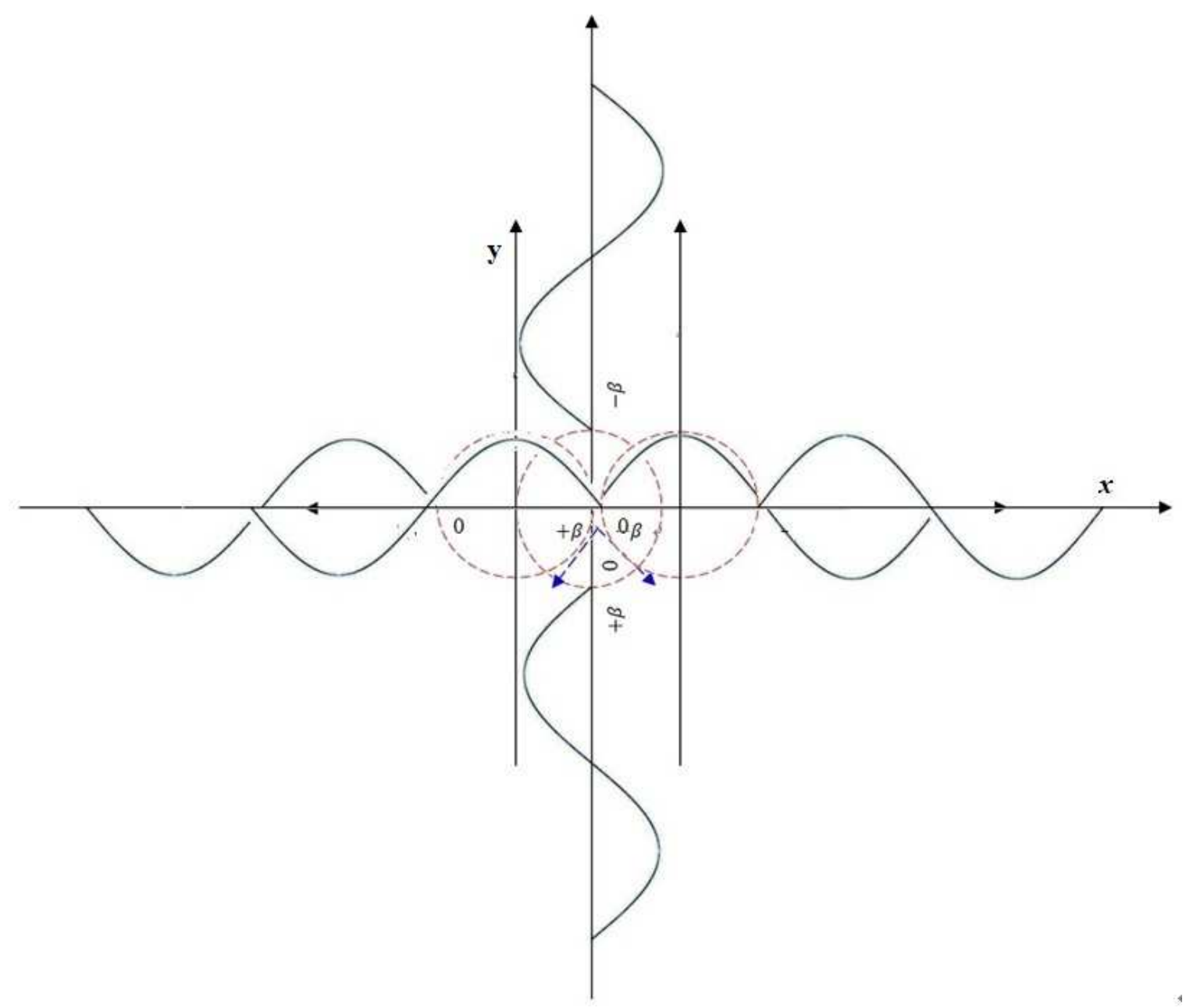

Figure 10. The normal distribution of joint Clustering - the nonlinear estimation interval of the central limit theorem.

The quasi thinking iterative programming and clustering (joint clustering) of heavy kernel clustering, the normal distribution of the weak robustness sliding angle depth feature information extraction of the discrete gradient data cluster the nonlinear estimation parameter space of the central limit theorem, and the weak distribution of the homomorphic structure of intelligent information such as dimension, wave shape and weak robustness sliding kernel. It has a complex variable circle (high-dimensional) logic split line sink system, which is composed of the nonlinear rotation of the center line source of the $\left(\sqrt{\operatorname{Cos}^{n}(A)}, i \cdot \sqrt{\operatorname{Sin}^{n}\left(A^{*}\right)}\right)$ notch and the source line sink wave [14]. 


$$
\begin{aligned}
& {\left[\begin{array}{ccc}
\operatorname{Cos}^{\frac{n}{2}}(A) & -\frac{\sigma_{\diamond}}{\sqrt{n}} \\
& A^{*} \leftrightarrow \bar{X}_{A} & \\
\frac{\sigma_{\diamond}^{*}}{\sqrt{n}} & & -i \cdot \operatorname{Sin}^{\frac{n}{2}}\left(A^{*}\right)
\end{array}\right] \rightarrow \frac{1}{4} \operatorname{Sin}\left(\left(P\left(\bar{X} \pm \frac{\sigma_{\diamond}}{\sqrt{n}} \mu_{1-\frac{\alpha}{2}}\right)+\frac{\pi}{4}\right)+2 k \pi\right) \text {, and } A^{*} \leftrightarrow \bar{X}_{A}=A^{*}, \bar{X}_{A}, \sigma_{\diamond} \in\left(\frac{\pi}{8}, \frac{\pi}{4}\right)} \\
& n=4 p, 8 p, \operatorname{Cos}^{\frac{n}{2}}(A) \otimes A^{*} \leftrightarrow \bar{X}_{A} \otimes\left(-i \cdot \operatorname{Sin}^{\frac{n}{2}}\left(A^{*}\right)\right), \frac{\sigma_{\bullet}^{*}}{\sqrt{n}} \otimes A^{*} \leftrightarrow \bar{X}_{A} \otimes\left(-\frac{\sigma_{\circ}}{\sqrt{n}}\right)
\end{aligned}
$$

The estimation interval of the central limit theorem of the high-dimensional complex variable circle normal distribution (multimode, periodic, oscillatory) with sample compression line is similar to that of the quasi thinking iterative programming of heavy kernel clustering [15].

$$
\begin{aligned}
& {\left[\begin{array}{ccc}
\operatorname{Cos}^{\frac{n}{2}}(A) & -\frac{\sigma_{\circ}}{\sqrt{n}} \\
& A^{*} \leftrightarrow \bar{X}_{A} & \\
\frac{\sigma_{\diamond}^{*}}{\sqrt{n}} & & -i \cdot \operatorname{Sin}^{\frac{n}{2}}\left(A^{*}\right)
\end{array}\right] \simeq\left(\frac{1}{4}\right)^{n}\left[\operatorname{Sin}\left(A_{1}+\sum_{i=2}^{m} A_{i}+n \cdot \frac{\pi}{4}\right)+\operatorname{Sin}\left(A_{1}^{*}-\sum_{i=2}^{m} A_{i}^{*}+n \cdot \frac{\pi}{4}\right)\right]^{n-1} \otimes\left(\operatorname{Cos}^{\frac{n}{2}}(A), i \cdot \operatorname{Sin}^{\frac{n}{2}}\left(A^{*}\right)\right),} \\
& \text { and if } \sigma_{\diamond}^{*}=i^{j} \sum_{i=2}^{2 N} i \cdot A_{j}^{*}, \sigma_{\diamond}=i^{j} \sum_{i=2}^{2 N} i \cdot \bar{X}_{A_{j}}, \\
& \left(\operatorname{Cos}^{\frac{n}{2}}(A), i \cdot \operatorname{Sin}^{\frac{n}{2}}\left(A^{*}\right)\right) \equiv 1, n=4 p, 8 p, A^{*} \leftrightarrow \bar{X}_{A} \text { is the sample compression line. }
\end{aligned}
$$

\subsection{The Normal Distribution (Multimodal, Periodic, Oscillatory) of the Center Source Limit Compression Line of the Image Entropy Kernel (Carcinoid)}

The normal distribution (multi peak, periodicity, oscillation) of the extreme compression line of the central source of the image entropy nucleus (carcinoid tumor). It can be seen that the other main reason for the change from inflammation of the image entropy nucleus to cancer is the degree of the compression line. From the medical point of view, it is the $D N A$ damage during the cell modification that causes the fluctuation, and the long-term damage will lead to the increase

$$
\sigma_{\diamond}^{*}=i^{j} \sum_{i=2}^{2 N} i \cdot A_{j}^{*}, \sigma_{\diamond}=i^{j} \sum_{i=2}^{2 N} i \cdot \bar{X}_{A_{j}}
$$

$$
\left[\begin{array}{ccc}
\operatorname{Cos}^{\frac{n}{2}}(A) & & -\frac{\sigma_{\circ}}{\sqrt{n}} \\
& A^{*} \leftrightarrow \bar{X}_{A} & \\
\frac{\sigma_{*}^{*}}{\sqrt{n}} & & -i \cdot \operatorname{Sin}^{\frac{n}{2}}\left(A^{*}\right)
\end{array}\right] \text {, and } n=8 p, \sigma_{\circ}^{*} \in\left(\frac{\pi}{8 p}, \frac{\pi}{4 p}\right), p \gg \Delta^{p}
$$

$A^{*} \leftrightarrow \bar{X}_{A}$ is the critical value of the compression line of high dimensional complex variable ball sampling.

The image entropy core of the compressed line of the high dimensional complex variable sphere sample is squeezed to the critical value [17]. The image entropy gliding nucleus is not constrained by $2 N+1$ lamination incision migration $\pi / 4$ hyperrefolding circular complex dynamic perturbation nucleus like fission, high dimensional refolding sphere compression line inner ring fragments, forming cancer cell source exfoliated cells.

$$
\Omega_{\text {Entropy kernel }}^{S=11}\left(O_{i p \cdot M \otimes M^{\prime}}^{\alpha \cdot \vec{n}}\right)=i \cdot C_{s} \cdot \sum_{K=4}^{N}\left[\left(\frac{\left(\vartheta_{11} \cdot \operatorname{Cos}\left(A_{1}^{*}+A_{2}^{*}\right)^{2 \nabla \cdot \frac{p}{q}}, i \cdot \frac{p}{q} \cdot \vartheta_{12} \cdot \operatorname{Sin}\left(\bar{X}_{1}^{A}+\bar{X}_{2}^{A}\right)^{2 \nabla \cdot \frac{p}{q}}\right)}{\lambda_{11} \cdot C_{s 1} \cdot\left(A_{j}^{* 2} \cdot \operatorname{Cos} A_{j}^{*}\right)^{\nabla}-\lambda_{12} \cdot C_{s 2} \cdot\left(\bar{X}_{j}^{A^{2}} \cdot \operatorname{Sin} \bar{X}_{j}^{A}\right)^{\nabla}}\right)\right]^{*}
$$




$$
\begin{gathered}
\text { or } i \cdot 2 C_{s} \cdot \frac{p}{q} \cdot \sum_{K=4}^{N}\left[\left(\frac{\left(\vartheta_{11} \cdot \operatorname{Cos}\left(i^{j} \sum_{i=1}^{2 N+1} i \cdot A_{j}^{*}\right), i \cdot \vartheta_{12} \cdot \operatorname{Sin}\left(i^{j} \sum_{i=2}^{2 N} i \cdot \bar{X}_{j}^{A}\right)\right)}{\lambda_{11} \cdot C_{s 1} \cdot\left(A_{j}^{* 2} \cdot \operatorname{Cos} A_{j}^{*}\right)^{\nabla}-\lambda_{12} \cdot C_{s 2} \cdot\left(\bar{X}_{j}^{A^{2}} \cdot \operatorname{Sin} \bar{X}_{j}^{A}\right)^{\nabla}}\right)\right]^{*} . \\
d^{2 K-1}\left(\operatorname{Cos}\left(A_{j}^{* K-2}\right) \otimes \operatorname{Sin}\left(\bar{X}_{j}^{A^{K-2}}\right)\right), \text { and } s=11 ; p, q=2,4,6, \ldots, 2 n
\end{gathered}
$$

\subsection{Fusion of Dumbbell Double Sphere Complex Sphere of Image Entropy Nucleus}

The fusion of dumbbell double sphere complex sphere of image entropy core, and the high dimensional double complex phere of limit compression line of central source. $A^{*} \leftrightarrow \bar{X}_{A} \rightarrow\left(\operatorname{Cos}\left(A_{1}^{*}, A_{2}^{*}\right)^{2 \nabla \cdot \frac{p}{q}}, i \cdot \operatorname{Sin}\left(\bar{X}_{1}^{A}, \bar{X}_{2}^{A}\right)^{2 \nabla \cdot \frac{p}{q}}\right)$ exchangeability of image entropy kernel $A_{1}^{*} \sim \bar{X}_{1}^{A}, A_{2}^{*} \sim \bar{X}_{2}^{A}[18]$.

$$
\left[\begin{array}{cc}
\operatorname{Cos}^{\frac{n}{2}}(A) & -\frac{\sigma_{\diamond}}{\sqrt{n}} \\
\frac{\sigma_{\diamond}^{*}}{\sqrt{n}} & \operatorname{Cos}\left(A_{1}^{*} \leftrightarrow \bar{X}_{2}^{A}\right)^{2 \nabla \cdot \frac{p}{q}}, i \cdot \operatorname{Sin}\left(A_{2}^{*} \leftrightarrow \bar{X}_{1}^{A}\right)^{2 \nabla \cdot \frac{p}{q}} \\
\text { and } \sigma_{\diamond}^{*}=i^{j} \sum_{i=2}^{2 N} i \cdot A_{j}^{*}, \sigma_{\diamond}=i^{j} \sum_{i=2}^{2 N} i \cdot \bar{X}_{A_{j}} \\
n \gg 8 p, \sigma_{\diamond}^{*} \in\left(\frac{\pi}{8 p}, \frac{\pi}{4 p}\right), p \gg \Delta^{2 p}, A_{1}^{*} \leftrightarrow \bar{X}_{2}^{A}, A_{2}^{*} \leftrightarrow \bar{X}_{1}^{A}
\end{array}\right]
$$

is the critical value from the sample compression line of the high dimensional double complex sphere to the fusion core

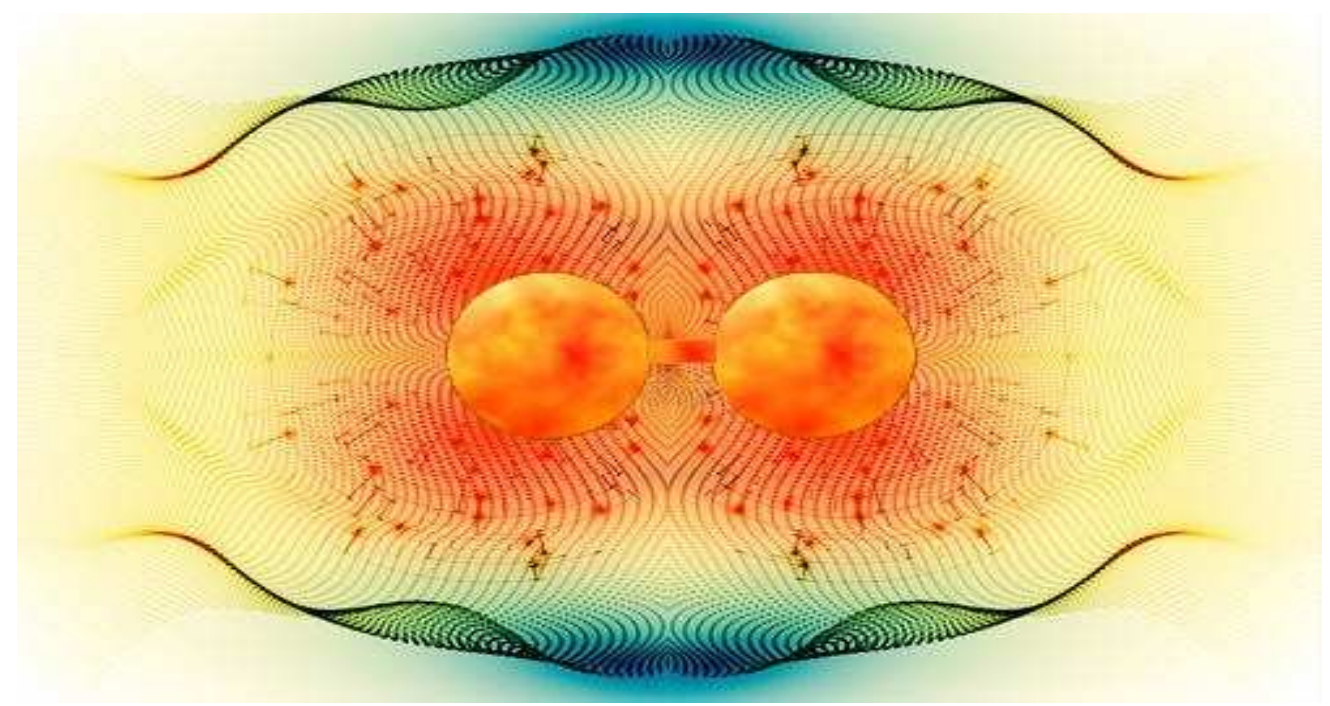

Figure 11. Image entropy core dumbbell double sphere complex variable sphere core.

For example, in renal cell carcinoma (left renal cell carcinoma) No. 1, the central limit compression line $A_{1}^{*} \leftrightarrow \bar{X}_{2}^{A}$, replicates (leads) the cancer cell DNA into the central limit compression line $A_{2}^{*} \leftrightarrow \bar{X}_{1}^{A}$ exchangeable core of No. 2 (right renal carcinoma) [19]. The unconstrained $2 N+1$ laminated notch will fragment the inner ring of the high dimensional complex variable sphere. The core of No. 1 and No. 2 nuclei began to collapse and form the exfoliated cells of cancer cells. And cancer cells and toxins spread rapidly [20].

For the renal cancer with image entropy nucleus dumbbell double sphere complex sphere, the normal distribution (multi peak, periodic, oscillatory) of the non compact compression line sink of the center source limit sampling of the

$$
\left(\operatorname{Cos}\left(A_{1}^{*} \leftrightarrow \bar{X}_{2}^{A}\right)^{2 \nabla \cdot \frac{p}{q}}, i \cdot \operatorname{Sin}\left(A_{2}^{*} \leftrightarrow \bar{X}_{1}^{A}\right)^{2 \nabla \cdot \frac{p}{q}}\right) \cong\left(\operatorname{Cos}\left(i^{j} \sum_{i=1}^{2 N+1} i \cdot A_{j}^{*}\right), i \cdot \operatorname{Sin}\left(i^{j} \sum_{i=2}^{2 N} i \cdot \bar{X}_{j}^{A}\right)\right)
$$


image entropy nucleus double sphere complex sphere kernel begins to relax; the non compact line sink of the high dimensional complex sampling begins to relax [21].

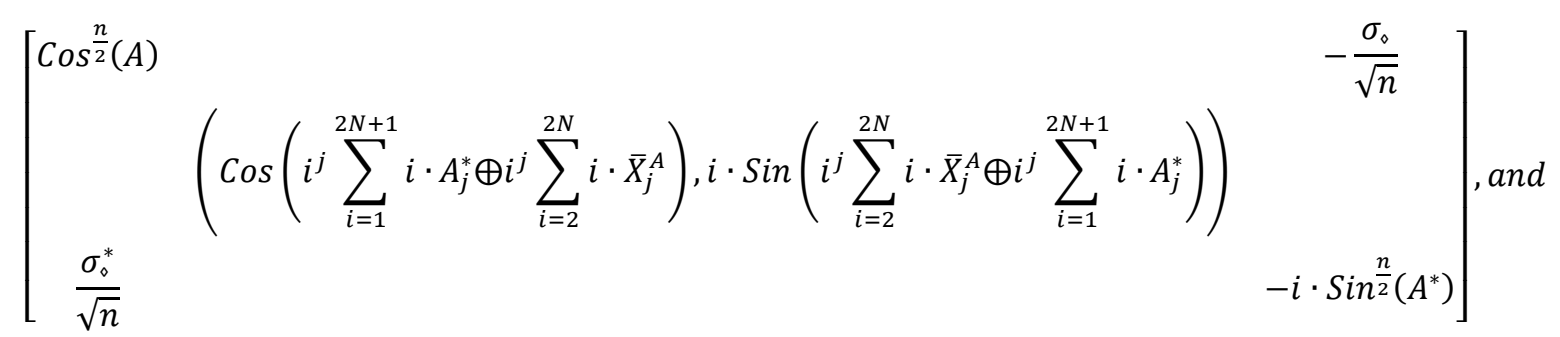$$
\sigma_{\diamond}^{*}=i^{j} \sum_{i=2}^{2 N} i \cdot A_{j}^{*}, \sigma_{\diamond}=i^{j} \sum_{i=2}^{2 N} i \cdot \bar{X}_{A_{j}}, n \leq 8 p, \sigma_{\diamond}^{*} \in\left(\frac{\pi}{8 p}, \frac{\pi}{4 p}\right), p \leq \Delta^{-p}
$$

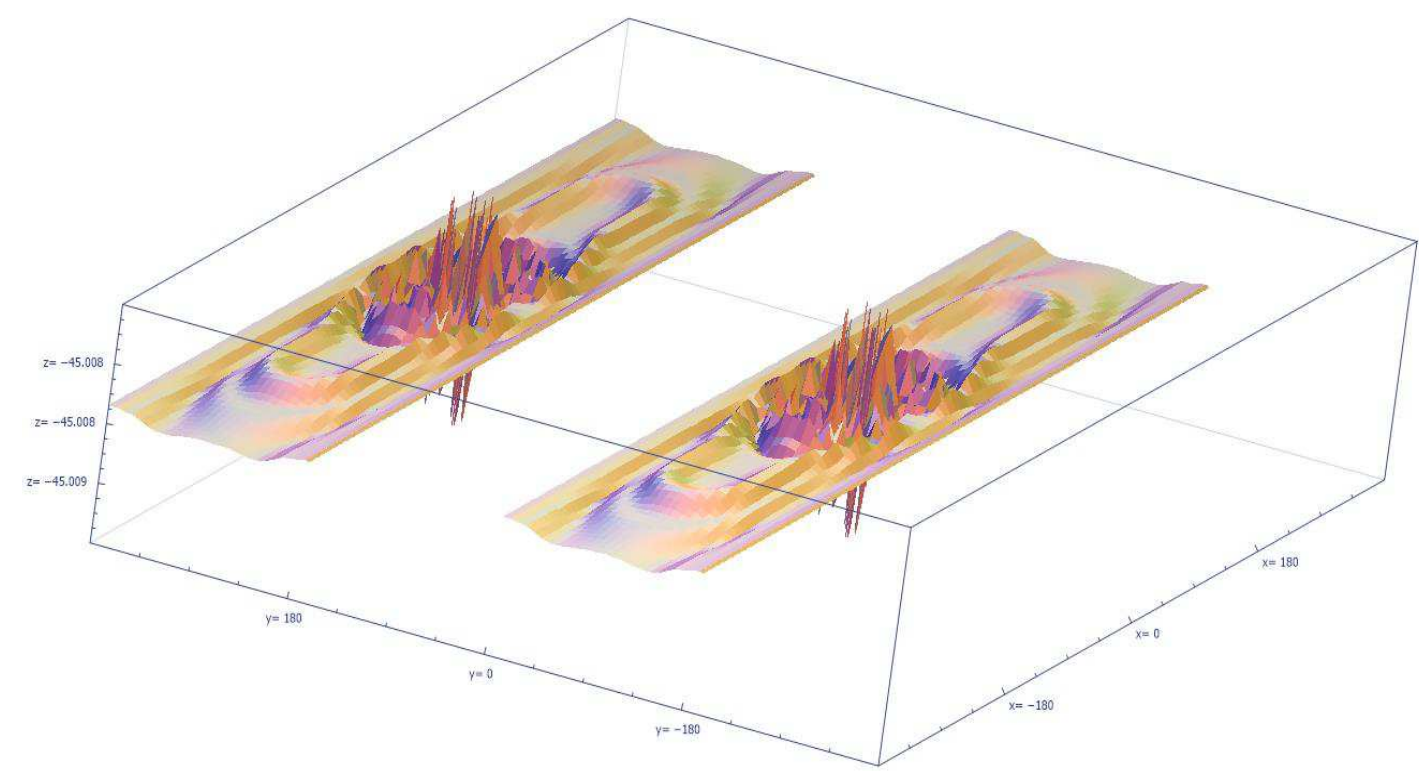

Figure 12. Image entropy kernel solitary wavelet of double sphere complex variable sphere kernel.

There is a weak nonlinear $\operatorname{Cos}^{\frac{8 \Delta^{-p}}{2}}(A), i \cdot \operatorname{Sin}^{\frac{8 \Delta^{-p}}{2}}\left(A^{*}\right)$-phase difference between the high-dimensional image of the growth trend of the intelligently generated nucleus of renal cancer cells and that of the normal cells modified nucleus [22].

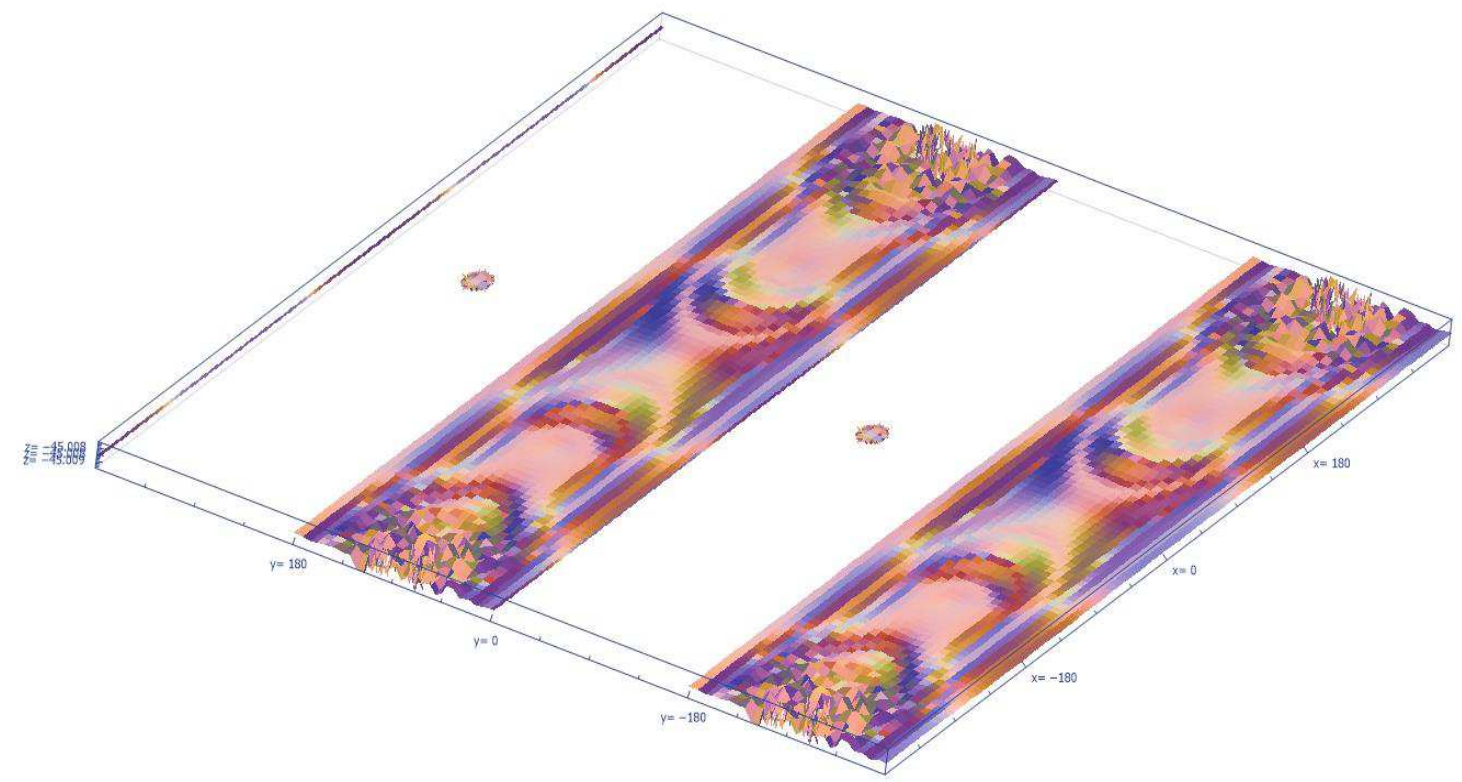

Figure 13. The growth trend of normal cell modification and damaged and repaired cancer cell bud nucleus. 
The kernel of dumbbell double sphere complex variable sphere with image entropy kernel. $\Omega_{\text {Entropy kernel }}^{S=11}\left(O_{\text {ip } \cdot M \otimes M^{\prime}}^{\alpha \cdot \vec{n}}\right) \rightarrow$ $\Omega_{\text {Entropy kernel }}^{s=10}\left(O_{i p \cdot M \otimes M^{\prime}}^{\propto \cdot \vec{n}}\right)$, Compression chord (compression line) of Hyper complex $p, s=11 \rightarrow 10$ wave causes release of exfoliated cells [23].

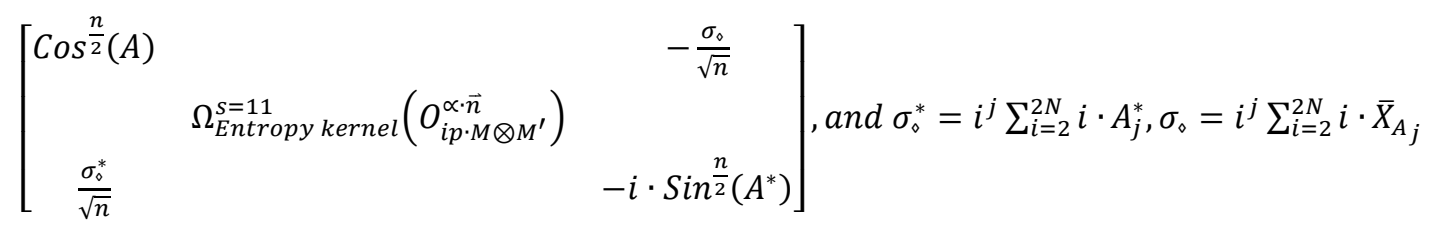

\section{Conclusion}

\subsection{The Center Source Superstring Is Compressed to Critical, and the Inner Ring of the High Dimensional Superstring Is Broken and Exfoliated Cells Are Produced}

The center source superstring is compressed to critical. The inner ring of high-dimensional superstring compression will be broken in the unrestrained $2 N+1$ stacking incision, and the number of exfoliated cells released will be calculated. Let's say that $A_{1}^{*}=1$ exfoliated cells, $\bar{X}_{1}^{A}$ cancer cells, and a unit of superstring is equal to $\left(\operatorname{Cos}\left(A_{1}^{*} \leftrightarrow \bar{X}_{2}^{A}\right)^{2 \nabla \cdot \frac{p}{q}}, i\right.$.

$\Omega_{\text {Entropy kernel }}^{S=11}\left(O_{i p \cdot M \otimes M^{\prime}}^{\propto \cdot \vec{n}}\right) \rightarrow \Omega_{\text {Entropy kernel }}^{S=10}\left(O_{i p \cdot M \otimes M^{\prime}}^{\propto \cdot \vec{n}}\right)=-i \cdot \frac{\pi+2 p_{11} \pi}{\sqrt{8 p_{11}}}+i \cdot \frac{\pi+2 p_{10} \pi}{\sqrt{8 p_{10}}}=-i \cdot \pi\left(\frac{23}{\sqrt{88}}-\frac{21}{\sqrt{80}}\right) \cong-0.326362$

Therefore, 32.6362 exfoliated cells were released from the 100 unit superstring of the image entropy kernel $\Omega_{\text {Entropy kernel }}^{S=11}\left(O_{i p \cdot M \otimes M^{\prime}}^{\alpha \cdot \vec{n}}\right) \rightarrow \Omega_{\text {Entropy kernel }}^{S=10}\left(O_{i p \cdot M \otimes M^{\prime}}^{\propto \cdot \vec{n}}\right)$.

\section{References}

[1] Zhu Rong Rong, Differential incremental equilibrium geometry protein granule Space folding, genome expression and cell modification, Fudan University, Volume 1, 2015-04-1: 1-112.

[2] Zhu Rong Rong, Differential incremental equilibrium geometry-protein granule Space folding, genome expression and cell modification General solution of nonlinear class of isolated wavelet -- effective nuclear trace information, Fudan University, Volume 2, 2015-04-11: 1-185.

[3] Zhu Rong Rong, Differential incremental equilibrium geometry - Effects of Cerebral Groove and Protein Granule Motion on Thinking Space and Mental Activity, 4-Dimensional Super-high-end Super-spherical Convex Spherical Fiber Cluster, Residual Product-like Cluster Petal Microfibers, Fudan University, Volume 3, 2015-04-18: 1-227.

[4] Lou Senyue, Tang Xiaoyan, Nonlinear Mathematical Physics Method, Beijing China, Science Press, 2006: 1-365.

[5] LABPHTEB M. A., Tria BAT B. B., Methods of Function of a Complex Variable Originally published in Russian under the title, 1956, 2006: 1-287.

[6] Zheng Weiwei, Complex Variable Function and Integral Transform, Northwest Industrial University Press, 2011: 1-396.
$\left.\operatorname{Sin}\left(A_{2}^{*} \leftrightarrow \bar{X}_{1}^{A}\right)^{2 \nabla \cdot \frac{p}{q}}\right)$

So how many exfoliated cells are released from $\Omega_{\text {Entropy kernel }}^{S=11}\left(O_{i p \cdot M \otimes M^{\prime}}^{\propto \cdot \vec{n}}\right) \rightarrow \Omega_{\text {Entropy kernel }}^{S=10}\left(O_{i p \cdot M \otimes M^{\prime}}^{\propto \cdot \vec{n}}\right)$, and how many exfoliated cells are released from 100 units of superstring [24].

\subsection{Image Entropy and Prediction of Future Development}

Whether the immune cells of human immune system are activated to intervene in inflammation, cancer and transition, this recognition puzzles life science researchers and medical researchers. Based on its internal logic, aidicom system uses the dieg algorithm to complete the detection and classification of lesions, as well as the prediction of future development.

[7] Zhang Wenxiu, Qiu Guofang, Uncertain Decision Making Based on Rough Sets, Beijing China, tsinghua university press, 2005: 1-255.

[8] Ren Fuyao, Complex Analytic Dynamic System, Shanghai China, Fudan University Press, 1996: 1-364.

[9] Zheng Jianhua, Meromorphic Functional Dynamics System, Beijing China, tsinghua university press, 2006: 1-413.

[10] C. Rogers W. K. Schief, Bäcklund and Darboux Transformations: Geometry and Modern Applications in Solition Theory, first published by Cambridge University, 2015: 1-292.

[11] W. Miller, Symmetry Group and Its Application, Beijing China, Science Press, 1981: 1-486.

[12] Gong Sheng, Harmonic Analysis on Typical Groups Monographs on pure mathematics and Applied Mathematics Number twelfth, Beijing China, Science Press, 1983: 1-314.

[13] Jari Kaipio Erkki Somersalo, Statistical and Computational Inverse Problems With 102 Figures, Spinger.

[14] Chen Zhonghu, Lie group guidance, Higher Education Press, 1997: 1-334.

[15] Gu Chaohao, Hu Hesheng, Zhou Zixiang, DarBoux Transformation in Solition Theory and Its Geometric Applications (The second edition), Shanghai science and technology Press, 1999, 2005: 1-271.

[16] Xiao Gang, Fibrosis of Algebraic Surfaces, Shanghai China, Shanghai science and technology Press, 1992: 1-180. 
[17] Qiu Chengtong, Sun Licha, Differential Geometry Monographs on pure mathematics and Applied Mathematics Number eighteenth, Beijing China, Science Press, 1988: 1-403.

[18] Ding Peizhu, Wang Yi, Group and its Express, Higher Education Press, 1999: 1-468.

[19] E. M. Chirka, Complex Analytic Sets Mathematics and Its Applications, Kluwer Academic Publishers Gerald Karp, Cell and Molecular Biology: Concepts and Experiments (3e), Higher Education Press, 2005: 1-792.

[20] Shou Tiande, Neurobiology, Higher Education Press, 2001, 2003: $1-470$.
[21] Su Jingcun, Topology of Manifold, Wuhan China, wuhan university press, 2005: 1-708.

[22] Shou Tiande, Neurobiology (2e), Higher Education Press, 2001, 2006: 1-548.

[23] Numerical Treatment of Multi-Scale Problems Porceedings of the $13^{\text {th }}$ GAMM-Seminar, Kiel, January 24-26, 1997 Notes on Numerical Fluid Mechanics Volume 70 Edited By Wolf Gang Hack Busch and Gabriel Wittum.

[24] Wu Chuanxi, Li Guanghan, Submanifold geometry, Beijing China, Science Press, 2002: 1-217. 